\section{Kynurenic acid accumulation underlies learning and memory impairment associated with aging}

\author{
Mihir Vohra, George A. Lemieux, Lin Lin, \\ and Kaveh Ashrafi
}

Department of Physiology, University of California at

San Francisco, San Francisco California 94158, USA

A general feature of animal aging is decline in learning and memory. Here we show that in Caenorhabditis elegans, a significant portion of this decline is due to accumulation of kynurenic acid (KYNA), an endogenous antagonist of neural $\mathrm{N}$-methyl-D-aspartate receptors (NMDARs). We show that activation of a specific pair of interneurons either through genetic means or by depletion of KYNA significantly improves learning capacity in aged animals even when the intervention is applied in aging animals. KYNA depletion also improves memory. We show that insulin signaling is one factor in KYNA accumulation.

Supplemental material is available for this article.

Received October 3, 2017; revised version accepted December 22, 2017.

Aging is the biggest risk factor for neurodegenerative diseases such as Alzheimer's disease, Huntington's disease, and Parkinson's disease, but learning declines with age even in the absence of other neurological pathologies (López-Otín et al. 2013; Wyss-Coray 2016). Few interventions are known to protect against age-induced learning impairment (Villeda et al. 2011; Dubal et al. 2014; Yu et al. 2017), and, even in these cases, the precise mechanisms by which age-dependent deficits are ameliorated remain unclear.

The kynurenine pathway $(\mathrm{KP})$ is an evolutionarily conserved pathway that catabolizes the essential amino acid tryptophan and produces several neuroactive compounds, including kynurenic acid (KYNA), a noncompetitive antagonist of $\mathrm{N}$-methyl-D-aspartate receptors (NMDARs) (Kessler et al. 1989). NMDARs are vital for learning in many organisms (Morris et al. 1986; Kano et al. 2008).

Caenorhabditis elegans exhibit associative learning, and the persistence of this association in the absence of further training is termed memory (Kauffman et al. 2010). Pairing of the odor butanone with food results in an enhanced attraction to this odor, a form of associative learning. Depending on the pairing regimen, this attraction is maintained for either short or long periods of time that are termed short-term or long-term associative

[Keywords: kynurenic acid; metabolism; learning; memory; aging; C. elegans]

Corresponding author: kaveh.ashrafi@ucsf.edu

Article published online ahead of print. Article and publication date are online at http://www.genesdev.org/cgi/doi/10.1101/gad.307918.117. memory, respectively (Kauffman et al. 2010). These processes use molecular components also required for mammalian learning or memory: The butanone learning paradigm depends on NMDARs, while long-term memory is dependent on cAMP response element-binding (CREB) protein (Kano et al. 2008; Kauffman et al. 2010; Amano and Maruyama 2011; Nishijima and Maruyama 2017; Vohra et al. 2017). Here, we investigate mechanisms that underlie learning and memory impairment associated with C. elegans aging.

\section{Results and Discussion}

Associative learning capacity undergoes a progressive ageinduced impairment in C. elegans (Kauffman et al. 2010). For example, 7 -day-old adult animals exhibit only $~ 5 \%$ of the learning capacity seen in day 1 adults (Supplemental Fig. S1A,B; Kauffman et al. 2010). Since many tissues deteriorate with age, it is possible that some irreparable damage underlies this impairment. However, simply increasing the duration of conditioning from 1 to $4 \mathrm{~h}$ is sufficient to counter, albeit partially, these learning impairments (Supplemental Fig. S1A,B). Thus, at least a portion of the age-onset deficits in plasticity is subject to improvements.

\section{KYNA accumulates with age and impairs learning}

Consistent with the role of KYNA as a noncompetitive antagonist of NMDARs and given that increasing the duration of conditioning confers a significant degree of learning capacity to aged animals, we considered the possibility that aberrant accumulation of KYNA may contribute to age-induced impairments. We found that elevating KYNA via mutation in $\mathrm{kmo-1}$ exacerbates ageinduced learning impairment, and depleting KYNA via mutation in nkat-1 is protective against it (Fig. 1A; Supplemental Fig. S1C). More importantly, reducing KYNA levels also slowed the rate of age-induced learning impairment, while increasing its levels exacerbated it (Supplemental Fig. S1D). Increasing the duration of conditioning could partially decelerate age-induced learning impairments in kmo-1 mutants but had no effect on the already improved learning capacity of nkat-1 mutants (Supplemental Fig. S1E,F). Importantly, altering KYNA levels does not affect $C$. elegans life span (Supplemental Fig. S1G; Vohra et al. 2017), indicating that the effects of this manipulation are not indirect consequences of extended life span. Although by day 7 of adulthood wildtype animals show almost no learning capacity upon $1 \mathrm{~h}$ of conditioning, their capacity for motility remains about the same as those of day 1 animals, and, as a population, they almost all remain alive. We also assessed naïve chemotaxis to different concentrations of butanone at various ages and found that it was unchanged regardless of KYNA levels (Supplemental Fig. S1H,I). Because chemotaxis to

(c) 2018 Vohra et al. This article is distributed exclusively by Cold Spring Harbor Laboratory Press for the first six months after the full-issue publication date (see http://genesdev.cshlp.org/site/misc/terms.xhtml). After six months, it is available under a Creative Commons License (Attribution-NonCommercial 4.0 International), as described at http:// creativecommons.org/licenses/by-nc/4.0/. 
A
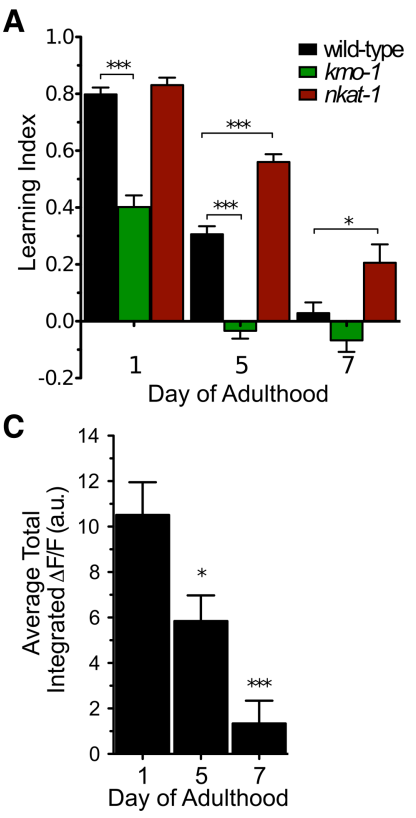

B

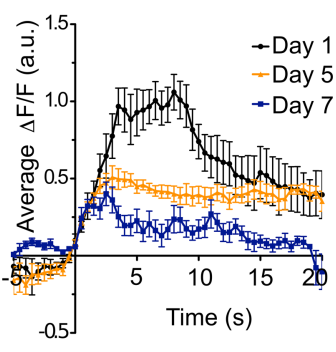

D

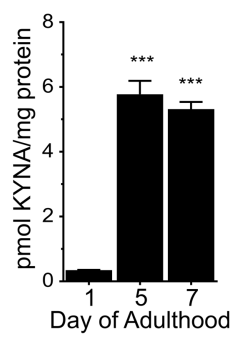

Figure 1. KYNA negatively modulates age-induced learning impairment. (A) Elevating KYNA (via kmo-1 mutation) exacerbates age-induced learning impairment, whereas depleting KYNA (via nkat-1 mutation) protects against it. $n=6-12 .\left(^{*}\right) P<0.05 ;\left(^{* * *}\right) P<0.001$ by two-way ANOVA (Bonferroni). See also Supplemental Figure S1. (B) Aging blunts the average intensity of spontaneous GCaMP transients in the RIM interneuron in wild-type animals. Transients from the 250 -sec imaging window are aligned to a -5 - to 20 -sec time axis. $n$ $=5$. $(C)$ Aging reduces the average total intensity of RIM GCaMP fluorescence in wild-type animals over the entire 250-sec imaging window. $n=5$. $\left({ }^{*}\right) \quad P<0.05 ; \quad(* *) \quad P<0.001$ by one-way ANOVA (Dunnett). (D) KYNA levels increase with age, as indicated by highperformance liquid chromatography (HPLC) measurements of whole-animal extracts. $\left.n=4-5 .{ }^{* * *}\right) P<0.0001$ by one-way ANOVA (Dunnett). All data are represented as mean \pm SEM.

butanone requires odor sensation and movement, this indicates that general sensorimotor circuits remain intact in aged animals and that the age-induced changes that we observed most likely represent impairment in plasticity.

In C. elegans, NMDARs are expressed in only a few pairs of neurons (Brockie et al. 2001). Among these, the activity of NMDARs in the RIM pair of interneurons is both sufficient and necessary for several types of learning behaviors, including butanone associative learning (Kano et al. 2008; Lau et al. 2013; Vohra et al. 2017). We observed previously that the activity of RIM neurons, as assessed by the intensity of spontaneous $\mathrm{Ca}^{2+}$ transients using GCaMP3.0 over an extended period of time $(250 \mathrm{sec})$, is a cellular correlate of learning such that conditions that enhance or diminish learning cause elevated or reduced RIM transients, respectively (Vohra et al. 2017). Thus, we investigated RIM transients upon conditioning as animals aged. To compare the average intensities of transients, we aligned transients from $5 \mathrm{sec}$ before their initiation to $20 \mathrm{sec}$ later. Consistent with the behavioral phenotype, the average intensity of RIM activity after conditioning decreased with age (Fig. 1B). Summation of the intensity of transients over the entire 250-sec imaging window demonstrated the same phenomenon, with no significant age-related changes to the number of transients observed during the imaging window (Fig. 1C). These findings suggested that KYNA accumulates

with age, dampening RIM activity and learning capacity. Indeed, when we extracted KYNA from animals, we found that levels increased significantly with age, as measured by high-performance liquid chromatography (HPLC) (Fig. 1D).

\section{Insulin signaling regulates KYNA production}

We next considered mechanisms that may underlie agedependent accumulation of KYNA. We first considered the possibility that this phenomenon may be due to retention of eggs by aging hermaphrodites. This was not the case, as a similar age-dependent impairment of learning capacity was noted in males (Supplemental Fig. S2A,B). We next considered the effects of insulin signaling, as this pathway regulates expression of KP enzymes (Zarse et al. 2012; Vohra et al. 2017). We sought to assess whether increasing insulin signaling beyond the levels seen in wild-type young animals has detrimental effects on learning. Insulin levels can be elevated by reduction-of-function mutations in the Bardet-Biedl syndrome gene homolog bbs-7 (also known as osm-12) (Lee et al. 2011) or the PTEN homolog daf-18 (Ogg and Ruvkun 1998). These mutants exhibited learning impairments at day 1 of adulthood that could be partially rescued by nkat-1 RNAi (Fig. 2A). There were no significant differences in naïve chemotaxis in bbs-7 or daf-18 mutants on control
A

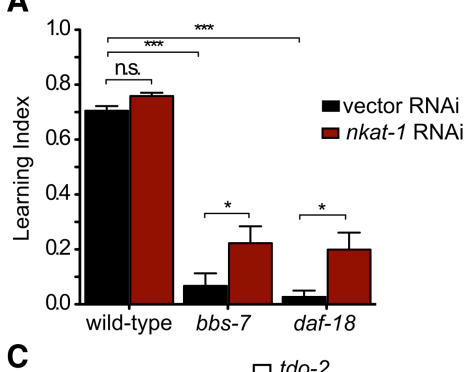

C

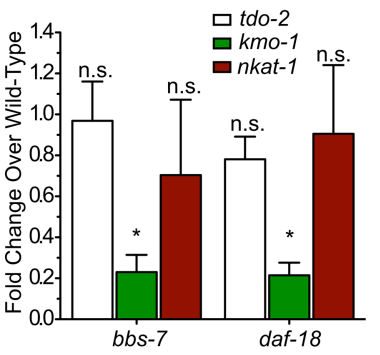

B

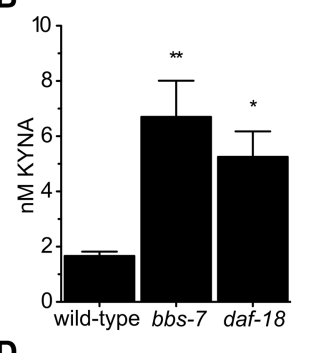

D

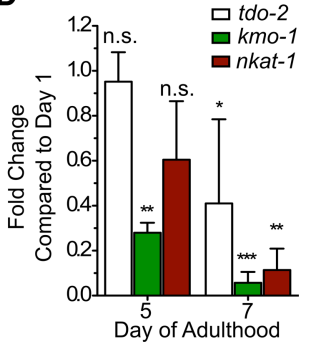

Figure 2. The effects of increased insulin signaling on learning and KYNA levels. (A) Animals with elevated insulin signaling have learning impairments even as young adults that can be partially rescued by nkat-1 RNAi. $\left.n=3-6 .\left(^{*}\right) P<0.05 ;{ }^{* * *}\right) P<0.001$ by two-way ANOVA (Bonferroni). See also Supplemental Figure S2. (B) Mutants with elevated insulin signaling have high KYNA levels, as indicated by HPLC measurements of whole-animal extracts. $n=3 .\left(^{*}\right) P<0.05$; $\left.{ }^{(* *}\right) P<0.01$ by one-way ANOVA (Dunnett). $(C)$ The change in transcript levels of KP genes in mutants with elevated insulin signaling as determined by real-time qPCR. Data are represented as fold change compared with wild type. $n=4$ biological replicates. $\left({ }^{*}\right) P<0.05$ by two-way ANOVA (Bonferroni). (D) The change in transcript levels of KP genes in wild-type animals at days 5 and 7 of adulthood as determined by real-time qPCR. Data are represented as fold change compared with wild type at day 1 of adulthood. $n=4$ biological replicates. $\left.\left.\left({ }^{*}\right) P<0.05 ;{ }^{* *}\right) P<0.01 ;{ }^{* * *}\right) P<0.001$ by two-way ANOVA (Bonferroni). All data are represented as mean \pm SEM. 
or nkat-1 RNAi (Supplemental Fig. S2C). KYNA levels in the $b b s-7$ and daf-18 mutants were high compared with wild-type animals (Fig. 2B).

To better understand how increases in insulin signaling contribute to increased accumulation of KYNA, we examined the expression patterns of genes that encode for various enzymes of the KP. We found that increasing insulin signaling decreased expression of kmo-1 (Fig. 2C), which competes with NKAT-1 for the same substrate (Supplemental Fig. S1C), and thus, as expected, its down-regulation results in accumulation of KYNA (Lemieux et al. 2015). Mimicking the effects of elevated insulin signaling, wild-type animals at days 5 and 7 of adulthood also had decreased expression of kmo-1 (Fig. 2D). These data suggest that insulin-mediated regulation of $k m o-1$ is likely a factor in KYNA production during the course of aging.

We next treated animals with daf-2 RNAi to impair insulin signaling and observed a deceleration of age-induced learning impairment (Supplemental Fig. S2D,E), consistent with results reported previously for daf-2(e1370) mutants at day 5 of adulthood (Kauffman et al. 2010). However, insulin signaling has broad effects, and daf-2 RNAi extends median life span (Supplemental Fig. S2F), making it difficult to discern whether the effects on ageinduced learning impairment are a result of specific changes in the circuitry responsible for learning. For a more specific approach, we reasoned that if KYNA accumulates with age and increasingly antagonizes neuronal function, then we should be able to bypass this accumulation and restore learning by artificially stimulating relevant neurons in the circuit. As RIM activity correlates with learning capacity in this paradigm, we chronically activated RIM neurons using a constitutively active protein kinase C (PKC) (Macosko et al. 2009). Similar to nkat-1 mutants, transgenic animals with activated RIM showed a deceleration of age-induced learning impairment (Fig. 3A; Supplemental Fig. S3A). Also similar to nkat-1 mutants, this manipulation affected plasticity without altering life span (Supplemental Fig. S3B).

\section{Initiation of KYNA depletion in aging adults counteracts} age-induced learning impairments

Since lifelong interventions such as nkat-1 mutation were effective at protecting against some of the age-induced learning impairments, we examined the effects of more acute interventions. In one strategy, we examined the effects of fasting on the learning capacity of aged animals. We found previously that a short (0.5- to 2-h) fast reduces KYNA levels in young animals (Lemieux et al. 2015; Vohra et al. 2017). Consistent with their overall elevated KYNA levels, longer periods of fasting (3-4 h) were needed for 5-d-old and 7-d-old animals to exhibit improved learning capacity (Fig. 3B; Supplemental Fig. S3C). Using HPLC, we confirmed that a 3 -h fast reduces KYNA levels in aged animals (Supplemental Fig. S3D).

In another strategy, we initiated nkat-1 RNAi beginning at day 3 of adulthood and examined its effects on the learning capacity of day 5 and day 7 adults. This intervention improved the learning capacity in aging animals to an extent similar to that seen in nkat-1 deletion mutants (Fig. 3C; Supplemental Fig. S3E). As expected, the effects of reducing KYNA levels on learning required NMDARs at every age (Supplemental Fig. S3F).
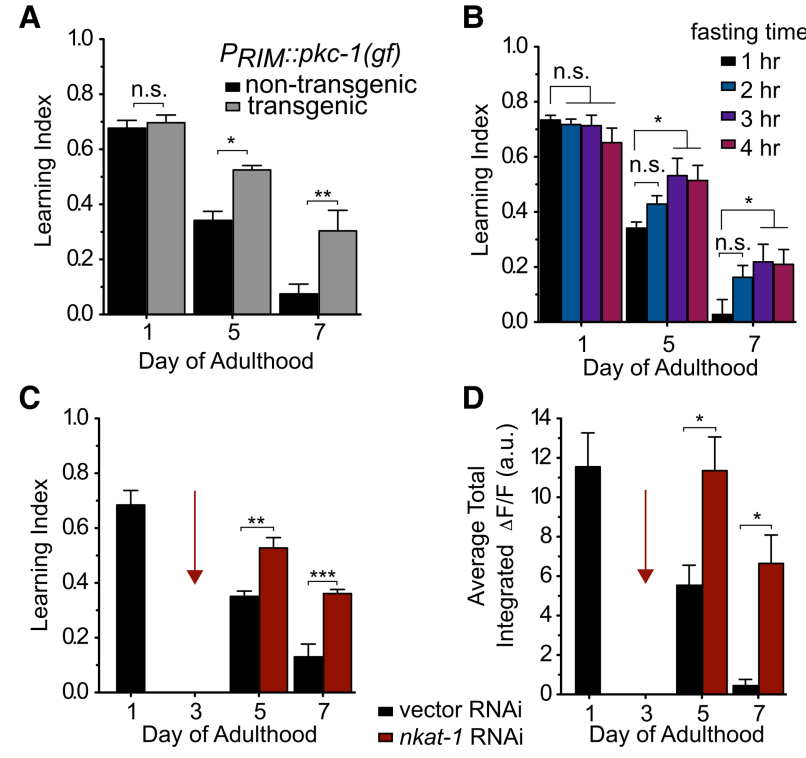

Figure 3. Adult depletion of KYNA protects against age-induced learning impairment. (A) Chronic activation of RIM by using a constitutively active PKC protects against age-induced learning impairment. $n=3-6$. (*) $P<0.05 ; \quad(* *) \quad P<0.01$ by two-way ANOVA (Bonferroni). See also Supplemental Figure S3. (B) Increasing fasting time before conditioning partially rescues age-induced learning impairment in wild-type animals. $n=3$. $\left({ }^{*}\right) P<0.05$ by two-way ANOVA (Bonferroni). Asterisks denote the statistical significance of a given condition compared with a 1-h fast. See also Supplemental Figure S3. (C) Depletion of KYNA in aging adults via nkat-1 RNAi beginning at day 3 of adulthood protects against age-induced learning impairment. The arrow indicates when nkat-1 RNAi was initiated. $n=3-$ 9. $\left(^{* *}\right) P<0.01 ;\left({ }^{* *}\right) P<0.001$ by two-way ANOVA (Bonferroni). See also Supplemental Figure S3. (D) Depletion of KYNA in aging adults via nkat-1 RNAi beginning at day 3 of adulthood allows for the reemergence of more robust $\mathrm{Ca}^{2+}$ transient intensity upon conditioning in aged animals. The average total intensity of RIM GCaMP fluorescence over the entire 250-sec imaging window in wild-type animals is shown. The arrow indicates when $n k a t-1$ RNAi was initiated. $n=5-7$. (*) $P<0.05$ by two-way ANOVA (Bonferroni). See also Supplemental Figure S3. All data are represented as mean \pm SEM.

While nkat-1 RNAi improved learning capacity to an extent similar to that of the nkat-1 deletion mutants and dependent on nmr-1, nkat-1 RNAi resulted in a marginally significant decrease of $17 \%$ in KYNA levels extracted from young animals and no statistically significant change in the highly elevated KYNA levels of day 7 adults (Supplemental Fig. S3G). We believe that the interpretation of these results should take into consideration the following findings: First, we found previously that deletion of nkat-1 reduced KYNA levels by $~ 50 \%$ (Lemieux et al. 2015). Other enzymatic activities-for example, that encoded by nkat-3-likely contribute to whole-animal KYNA pools. Thus, it is not unexpected that knocking down of nkat-1 would result in a more modest change in KYNA levels compared with nkat-1 mutants. Second, within the nervous system, nkat-1 is expressed in only a few neurons, and neuron-specific reconstitutions showed previously that expression of nkat1 only in RIM is sufficient to account for the effects of KYNA on learning (Vohra et al. 2017). Similarly, reconstitution of nmr-1 in RIM alone was found to be sufficient for learning (Vohra et al. 2017). Together, these findings strongly suggest that the local production of KYNA in 
RIM rather than the total organismal production of KYNA is most relevant to influencing this learning paradigm. Consistent with this, we found that while extended fasting in day 7 animals reduced KYNA levels (Supplemental Fig. S3D) and improved performance in the learning assay (Fig. 3B), on an absolute basis, the overall levels of KYNA in the 3 -h-fasted day 7 animals still vastly exceeded those of day 1 animals (Fig. 1D). Thus, the whole-animal extraction assay has only a limited capacity for revealing the local changes in KYNA, which is most relevant to learning capacity.

It is not currently experimentally feasible to directly measure KYNA levels local to RIM neurons. A readout that is functionally relevant to learning and can serve as a close proxy of localized levels of KYNA is measurements of $\mathrm{Ca}^{2+}$ levels in RIM. We found that, congruent with behavioral effects, nkat-1 RNAi initiated at day 3 resulted in enhanced $\mathrm{Ca}^{2+}$ readouts from RIM in day 5 and day 7 animals (Fig. 3D; Supplemental Fig. S3H). Thus, strategies that deplete KYNA locally to RIM can be effective in improving learning capacity despite overall elevated KYNA levels.

\section{KYNA levels affect memory}

While learning is a prerequisite for memory, in some cases, learning and memory can be attributed to distinct molecular mechanisms. For instance, CREB as well as many CREB-induced genes associated with synaptic transmission are required for long-term memory to butanone but not for the formation of associative learning or shortterm memory (Kauffman et al. 2010; Lakhina et al. 2015). In contrast, some mechanisms, such as AMPAR function, are required for both learning and memory (Kauffman et al. 2010). Thus, we sought to determine whether KYNA levels also modulate memory. In a shortterm associative memory assay, animals were conditioned with food and butanone and subsequently given food in the absence of butanone. Short-term memory in day 1 adult wild-type animals lasted for $\sim 90 \mathrm{~min}$ after conditioning, while kmo-1 mutants (which have high KYNA) exhibited accelerated deterioration of this memory, and nkat-1 mutants (which have low KYNA) had improved memory maintenance (Fig. 4A). The improvement seen upon loss of nkat-1 on short-term memory was also seen as animals aged, as exemplified by examination of day 5 adults (Supplemental Fig. S4A).

Next, we tested the effects of KP metabolites in a longterm memory assay where, instead of a single conditioning period, animals were alternatingly conditioned and fasted seven times (Kauffman et al. 2010). Maintenance of memory in young wild-type animals was largely extinguished by $\sim 40 \mathrm{~h}$ (Fig. 4B). Memory loss was accelerated in kmo-1 mutants, but there was very little loss at $40 \mathrm{~h}$ in nkat-1 mutants (Fig. 4B). Moreover, the enhancement in long-term memory performance of nkat-1 mutants also extended to day 3 and day 5 animals (Supplemental Fig. S4B).

We confirmed that the effects of KYNA deficiency on long-term memory are entirely NMDAR-dependent (Fig. 4C). Next, we examined the requirement for crh-1, which encodes for the transcription factor CREB (Kauffman et al. 2010). While RNAi against crh-1 did not affect the initial formation of the learned association to butanone, it degraded the ability of nkat-1 mutants to maintain long-
A

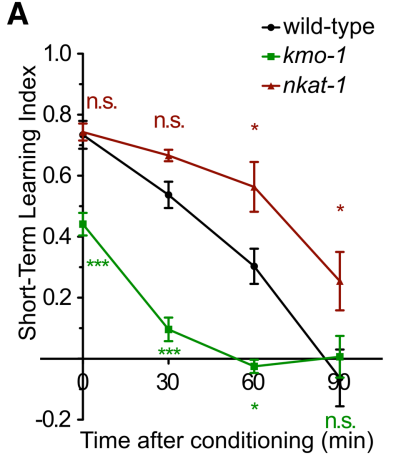

C
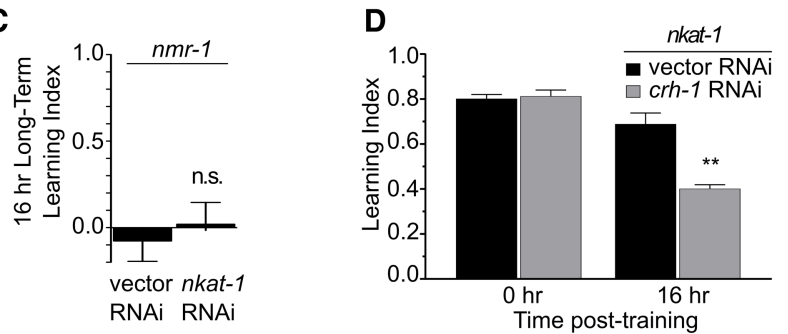

Figure 4. KYNA negatively modulates short-term and long-term memory. (A) Elevating KYNA (via inactivation of $\mathrm{kmo}-1$ ) impairs short-term memory in day 1 adults, whereas depleting KYNA (via inactivation of nkat-1) enhances it. $n=3 .\left(^{*}\right) P<0.05$; $\left(^{* * *}\right) P<0.001$ by two-way ANOVA (Bonferroni). Asterisk colors denote statistical significance of a given strain compared with wild type. See also Supplemental Figure S4. (B) Elevating KYNA impairs long-term memory in day 1 adults, whereas depleting KYNA enhances it. $n=$ 3. $\left(^{* *}\right) P<0.01 ;\left({ }^{* * *}\right) P<0.001$ by two-way ANOVA (Bonferroni). Asterisk colors denote the statistical significance of a given strain compared with wild type. See also Supplemental Figure S4. (C) $n m r-1$ mutation impairs 16-h long-term memory in day 1 adults and cannot be rescued by KYNA depletion. $n=3$, significance measured by twotailed $t$-test. $(D)$ Knockdown of $c r h$-1 blunts 16-h long-term memory but not the formation of the learned association in day $1 \mathrm{nkat}-1 \mathrm{mu}-$ tant adults. $n=3 .\left(^{* *}\right) P<0.01$ by two-tailed $t$-test compared with agematched vector control. All data are represented as mean \pm SEM.

term associative memory (Fig. 4D). Since increasing CREB levels enhances long-term associative memory (Kauffman et al. 2010), these data raise the possibility that KYNA levels modulate CREB activity, although how this modulation may be achieved is currently unknown. It is also currently unclear whether loss of KYNA improves memory only through its enhancements of learning. Potential involvement of other processes is suggested by the finding that while day 1 wild type and nkat-1 mutants achieve similarly robust associations, nkat-1 mutants still retain the association better (Fig. 4B).

In summary, we found that a portion of the age-dependent decline in a rudimentary form of neural plasticity in C. elegans is subject to manipulation by changes in KYNA levels. Our findings indicate that accumulation of KYNA accounts for the portion of the age-dependent decline in learning that can be counteracted by interventions such as fasting. This is consistent with the proposed role of KYNA as a competitive antagonist of NMDARs. Additionally, we found that loss of KYNA improves the maintenance of memory in both short-term and longterm memory paradigms. While it remains unknown why KYNA accumulates with age, our findings suggest that elevated insulin signaling is at least one factor that drives it. Thus, age-related accumulation of KYNA may 
not be a program per se but a consequence of various physiological changes-including elevated insulin signalingthat collectively affect flux through the $\mathrm{KP}$, resulting in KYNA accumulation.

There is an intriguing correlation between conditions or treatments that impact life span generally (and neural health more specifically) and levels of KYNA. For example, conditions that are generally considered detrimental to aging and neural functions such as inflammation and elevated insulin signaling are associated with accumulation of KP metabolites (Agudelo et al. 2014; Cervenka et al. 2017), while beneficial treatments such as dietary restriction reduce KYNA (Lemieux et al. 2015; Vohra et al. 2017). Each of the detrimental and beneficial regimens is certain to exert its effects through a multitude of processes; nevertheless, KYNA is emerging as a key link between each of these interventions and neural functions. Importantly, modulation of KYNA does not appear to affect life span itself; thus, its effects on neural functions are specific and not simply consequences of overall improved health. Our data also indicate that while accumulation of KYNA significantly contributes to age-onset learning impairments, it is not the only reason for this decline. After all, even the treatments that have the most robust effects on animal life span do not prevent aging-they simply delay onset of various impairments or extend median and maximum life spans.

The importance of KYNA production for learning and memory is likely to be relevant in other species as well, given that changes in KYNA levels are associated with many neurodegenerative disorders in humans such as Parkinson's disease (Ogawa et al. 1992), Alzheimer's disease (Guillemin et al. 2005; Bonda et al. 2010), and Huntington's disease (Beal et al. 1992; Pearson and Reynolds 1992). The biggest risk factor for these diseases is age, and, consistent with our findings that KYNA accumulates with age in C. elegans, it is reported to accumulate in rat brains as well (Moroni et al. 1988; Gramsbergen et al. 1992). There has long been an appreciation for the link between metabolism and neurological decline, and our findings suggest that KYNA may be a major mechanism by which this communication occurs. Our ability to decrease KYNA production in aging adults to decelerate age-induced learning impairment provides encouraging evidence that interventions later in life may be effective in ameliorating neurological decline.

\section{Materials and methods}

Strains

Strains used for this study are listed in the Supplemental Material.

\section{Materials}

Chemicals were purchased from Sigma. HT-115 Escherichia coli encoding double-stranded RNAi were from existing libraries (Dillin et al. 2002; Kamath et al. 2003). Animals were placed on RNAi bacteria at the first larval stage unless specified otherwise.

\section{Butanone associative learning and memory}

Butanone associative learning and memory assays were performed as described previously (Kauffman et al. 2010). Synchronized animals were cultivated, fasted, conditioned, and allowed to chemotax at $20^{\circ} \mathrm{C}$. At day 1 of adulthood, animals were either assayed or transferred to plates with $100 \mu \mathrm{M}$ FUDR to prevent the development of progeny and then assayed at day 5 or 7 of adulthood. The conditioning period was $1 \mathrm{~h}$, and the longer conditioning period used in Supplemental Figure S1, A and $\mathrm{B}$, was $4 \mathrm{~h}$.

\section{Culture of C. elegans for metabolite determination}

Synchronized L1 animals were grown in $15 \mathrm{~mL}$ of liquid cultures of 20,000 animals in S-medium and OP50 bacteria at an OD of 9 and shaken at 150 $\mathrm{rpm}$ at $20^{\circ} \mathrm{C}$. In the case of RNAi experiments, overnight cultures of HT115 RNAi bacteria in LB broth containing $100 \mu \mathrm{g} / \mathrm{mL}$ ampicillin were induced with $4 \mathrm{mM}$ IPTG for $2 \mathrm{~h}$ before concentration and resuspension in S-medium containing the same concentrations of ampicillin and IPTG, and then age-synchronized C. elegans were added. At the L4 stage, animals were collected, placed at $4^{\circ} \mathrm{C}$, washed three times in S-basal, washed once in potassium citrate buffer $(10 \mathrm{mM}$ potassium citrate at $\mathrm{pH} 6.0,100 \mathrm{mM}$ $\mathrm{NaCl}$ ), frozen in liquid nitrogen, and placed at $-80^{\circ} \mathrm{C}$ until metabolite extraction. For metabolite determination of aging adults, L4 animals were transferred to S-medium and bacteria that contained $100 \mu \mathrm{M}$ FUDR to prevent the development of progeny. Beginning on day 1 of adulthood, animals were transferred to new $25 \mathrm{~mL}$ of cultures of medium and bacteria every other day to prevent starvation until they were collected for extraction. Adult cultures were washed once with S-basal, and then viable adults were purified by flotation on ice-cold $30 \%$ sucrose and washed once with cold S-basal and twice with cold potassium citrate buffer before freezing.

\section{Metabolite extraction and determination}

Metabolite extraction and determination were performed as described previously (Lemieux et al. 2015).

\section{Real-time $q P C R$}

RNA extraction, cDNA preparation, and real-time qPCR were performed as described previously (Van Gilst et al. 2005) from four biological replicates of each condition. Data were standardized to actin (act-1), and primer sequences are listed in Supplemental Table S1.

\section{Statistical analyses}

In all graphs, error bars represent mean + SEM. One-way or two-way ANOVAs were used for comparisons between multiple conditions, and appropriate post-tests were used. When all conditions were compared with one control, a Dunnett correction was used. When conditions were compared among themselves, a Bonferroni correction was used.

\section{Acknowledgments}

We thank members of the Ashrafi laboratory for discussions and comments on the manuscript. K.A. was supported by National Institutes of Health (NIH) R01AG046400 and the Program in Breakthrough Biomedical Research. M.V. was supported by NIH R01AG011816, the Hillblom Foundation Graduate Student Fellowship, the Genentech Foundation Predoctoral Fellowship, and the Glenn/AFAR (American Federation for Aging Research) Aging Research Scholarship.

Author contributions: M.V., G.A.L., and K.A. designed experiments. M. V., G.A.L., and L.L. performed the experiments. M.V., G.A.L., and K.A. analyzed the data and wrote the manuscript.

\section{References}

Agudelo LZ, Femenía T, Orhan F, Porsmyr-Palmertz M, Goiny M, Martinez-Redondo V, Correia JC, Izadi M, Bhat M, Schuppe-Koistinen I, et al. 2014. Skeletal muscle PGC-1al modulates kynurenine metabolism and mediates resilience to stress-induced depression. Cell 159: 33-45.

Amano H, Maruyama IN. 2011. Aversive olfactory learning and associative long-term memory in Caenorhabditis elegans. Learn Mem 18: 654-665. 
Beal MF, Matson WR, Storey E, Milbury P, Ryan EA, Ogawa T, Bird ED. 1992. Kynurenic acid concentrations are reduced in Huntington's disease cerebral cortex. J Neurol Sci 108: 80-87.

Bonda DJ, Mailankot M, Stone JG, Garrett MR, Staniszewska M, Castellani RJ, Siedlak SL, Zhu X, Lee H, Perry G, et al. 2010. Indoleamine 2,3-dioxygenase and 3-hydroxykynurenine modifications are found in the neuropathology of Alzheimer's disease. Redox Rep 15: 161-168.

Brockie PJ, Madsen DM, Zheng Y, Mellem J, Maricq AV. 2001. Differential expression of glutamate receptor subunits in the nervous system of Caenorhabditis elegans and their regulation by the homeodomain protein UNC-42. I Neurosci 21: 1510-1522.

Cervenka I, Agudelo LZ, Ruas JL. 2017. Kynurenines: tryptophan's metabolites in exercise, inflammation, and mental health. Science 357: eaaf9794.

Dillin A, Crawford DK, Kenyon C. 2002. Timing requirements for insulin/ IGF-1 signaling in C. elegans. Science 298: 830-834.

Dubal DB, Yokoyama JS, Zhu L, Broestl L, Worden K, Wang D, Sturm VE, Kim D, Klein E, Yu G-Q, et al. 2014. Life extension factor klotho enhances cognition. Cell Rep 7: 1065-1076.

Gramsbergen JB, Schmidt W, Turski WA, Schwarcz R. 1992. Age-related changes in kynurenic acid production in rat brain. Brain Res 588: 1-5.

Guillemin GJ, Brew BI, Noonan CE, Takikawa O, Cullen KM. 2005. Indoleamine 2,3 dioxygenase and quinolinic acid immunoreactivity in Alzheimer's disease hippocampus. Neuropathol Appl Neurobiol 31: 395-404.

Kamath RS, Fraser AG, Dong Y, Poulin G, Durbin R, Gotta M, Kanapin A, Le Bot N, Moreno S, Sohrmann M, et al. 2003. Systematic functional analysis of the Caenorhabditis elegans genome using RNAi. Nature 421: 231-237.

Kano T, Brockie PJ, Sassa T, Fujimoto H, Kawahara Y, Iino Y, Mellem JE, Madsen DM, Hosono R, Maricq AV. 2008. Memory in Caenorhabditis elegans is mediated by NMDA-type ionotropic glutamate receptors. Curr Biol 18: 1010-1015.

Kauffman AL, Ashraf JM, Corces-Zimmerman MR, Landis JN, Murphy CT. 2010. Insulin signaling and dietary restriction differentially influence the decline of learning and memory with age. PLoS Biol 8: e1000372.

Kessler M, Terramani T, Lynch G, Baudry M. 1989. A glycine site associated with $\mathrm{N}$-methyl-D-aspartic acid receptors: characterization and identification of a new class of antagonists. J Neurochem 52: 13191328.

Lakhina V, Arey RN, Kaletsky R, Kauffman A, Stein G, Keyes W, Xu D, Murphy CT. 2015. Genome-wide functional analysis of CREB/longterm memory-dependent transcription reveals distinct basal and memory gene expression programs. Neuron 85: 330-345.

Lau HL, Timbers TA, Mahmoud R, Rankin CH. 2013. Genetic dissection of memory for associative and non-associative learning in Caenorhabditis elegans. Genes Brain Behav 12: 210-223.
Lee BH, Liu I, Wong D, Srinivasan S, Ashrafi K. 2011. Hyperactive neuroendocrine secretion causes size, feeding, and metabolic defects of $C$. elegans Bardet-Biedl syndrome mutants. PLoS Biol 9: e1001219.

Lemieux GA, Cunningham KA, Lin L, Mayer F, Werb Z, Ashrafi K. 2015. Kynurenic acid is a nutritional cue that enables behavioral plasticity. Cell 160: 119-131.

López-Otín C, Blasco MA, Partridge L, Serrano M, Kroemer G. 2013. The hallmarks of aging. Cell 153: 1194-1217.

Macosko EZ, Pokala N, Feinberg EH, Chalasani SH, Butcher RA, Clardy J, Bargmann CI. 2009. A hub-and-spoke circuit drives pheromone attraction and social behaviour in C. elegans. Nature 458: 1171-1175.

Moroni F, Russi P, Carlá V, Lombardi G. 1988. Kynurenic acid is present in the rat brain and its content increases during development and aging processes. Neurosci Lett 94: 145-150.

Morris RG, Anderson E, Lynch GS, Baudry M. 1986. Selective impairment of learning and blockade of long-term potentiation by an N-methyl-Daspartate receptor antagonist, AP5. Nature 319: 774-776.

Nishijima S, Maruyama IN. 2017. Appetitive olfactory learning and longterm associative memory in Caenorhabditis elegans. Front Behav Neurosci 11: 80.

Ogawa T, Matson WR, Beal MF, Myers RH, Bird ED, Milbury P, Saso S. 1992. Kynurenine pathway abnormalities in Parkinson's disease. Neurology 42: 1702-1706.

Ogg S, Ruvkun G. 1998. The C. elegans PTEN homolog, DAF-18, acts in the insulin receptor-like metabolic signaling pathway. Mol Cell 2: 887-893.

Pearson SJ, Reynolds GP. 1992. Increased brain concentrations of a neurotoxin, 3-hydroxykynurenine, in Huntington's disease. Neurosci Lett 144: $199-201$.

Van Gilst MR, Hadjivassiliou H, Jolly A, Yamamoto KR. 2005. Nuclear hormone receptor NHR-49 controls fat consumption and fatty acid composition in C. elegans. PLOS Biol 3: e53.

Villeda SA, Luo J, Mosher KI, Zou B, Britschgi M, Bieri G, Stan TM, Fainberg N, Ding Z, Eggel A, et al. 2011. The ageing systemic milieu negatively regulates neurogenesis and cognitive function. Nature 477: 90-94.

Vohra M, Lemieux GA, Lin L, Ashrafi K. 2017. The beneficial effects of dietary restriction on learning are distinct from its effects on longevity and mediated by depletion of a neuroinhibitory metabolite. PLOS Biol 15: e2002032.

Wyss-Coray T. 2016. Ageing, neurodegeneration and brain rejuvenation. Nature 539: 180-186.

Yu X-W, Ii DMC, Oh MM, Yin JC, Disterhoft JF. 2017. CREB overexpression in dorsal CA1 ameliorates long-term memory deficits in aged rats. Elife 6: e19358.

Zarse K, Schmeisser S, Groth M, Priebe S, Beuster G, Kuhlow D, Guthke R, Platzer M, Kahn CR, Ristow M. 2012. Impaired insulin/IGF1 signaling extends life span by promoting mitochondrial L-proline catabolism to induce a transient ROS signal. Cell Metab 15: 451-465. 


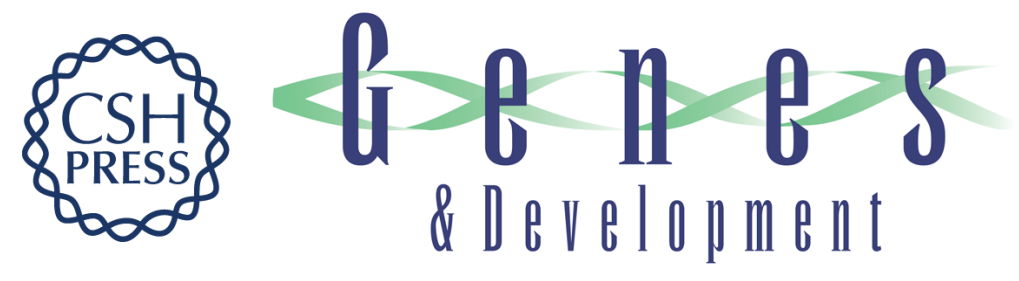

\section{Kynurenic acid accumulation underlies learning and memory impairment associated with aging}

Mihir Vohra, George A. Lemieux, Lin Lin, et al.

Genes Dev. 2018, 32: originally published online January 31, 2018

Access the most recent version at doi:10.1101/gad.307918.117

\section{Supplemental http://genesdev.cshlp.org/content/suppl/2018/01/31/gad.307918.117.DC1 Material}

References This article cites 32 articles, 4 of which can be accessed free at: http://genesdev.cshlp.org/content/32/1/14.full.html\#ref-list-1

Creative This article is distributed exclusively by Cold Spring Harbor Laboratory Press for the first Commons six months after the full-issue publication date (see

License http://genesdev.cshlp.org/site/misc/terms.xhtml). After six months, it is available under a Creative Commons License (Attribution-NonCommercial 4.0 International), as described at http://creativecommons.org/licenses/by-nc/4.0/.

Email Alerting Receive free email alerts when new articles cite this article - sign up in the box at the top Service right corner of the article or click here.

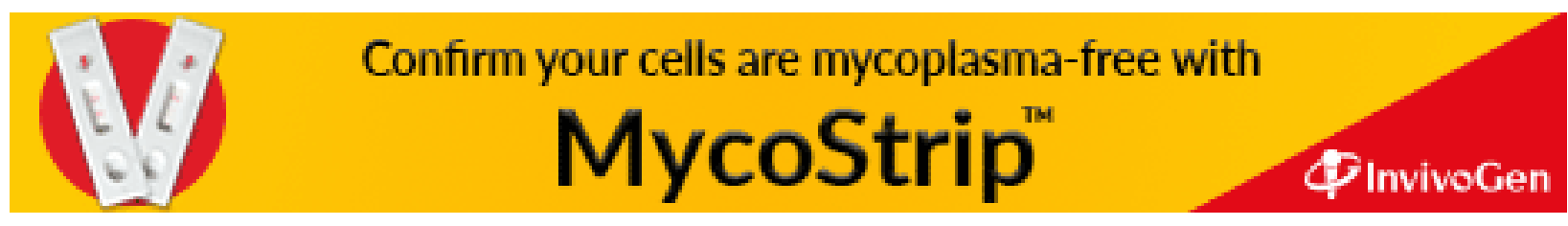

